implementation of a 2 hour point-of-care (POC) STI care model for clinical trial participants.

Methods As part of a Phase I/IIa vaccine study, CAPRISA implemented POC STI testing for chlamydia, gonorrhoea (Xpert CT/NG, $90 \mathrm{~min}$ ) and trichomonas (OSOM, $15 \mathrm{~min}$ ) at a site laboratory of a research clinic in Durban, South Africa, in July 2015. Since then, the POC model has been adopted by 5 other prevention studies, becoming the main STI testing model at CAPRISA.

Results A total of 1426 Xpert CT/NG assays were run on two 4-module Genexpert machines between July 2015 and November 2016. Chlamydia was detected in 206 (14.4\%), gonorrhoea in $79(5.5 \%)$ samples, and 52 (3.6\%) samples showed both infections. No infection was detected in 1070 (75.0\%) samples. $143(8.6 \%)$ samples showed either an error message $(5.0 \%)$, an invalid result $(3.0 \%)$ or no result $(0.6 \%)$ requiring repeat testing, either on the same or next convenient visit. Trichomonas testing was conducted simultaneously on all female participants $(n=1093$, prevalence $2.7 \%)$. Gram staining to diagnose bacterial vaginosis and candida infection was performed on-site in one of the studies. Implementation of this model allowed early detection of screen failures for Phase I/ IIa vaccine studies. During follow-up, participants with STIs received enhanced risk reduction counselling and immediate treatment on the day of sample collection. This resulted in improved care, early partner notification, and cost-savings by avoiding unnecessary screening procedures and repeat visits.

Conclusion A 2 hour POC STI testing model can streamline screening and follow-up of participants in HIV prevention studies, and should be considered for implementation by other research sites.

Support: Cepheid loaned two 4-module Genexpert machines to the study team free-of-charge, but did not contribute to the preparation of this abstract

\section{P3.110 HOW SHOULD WE MONITOR CHLAMYDIA CONTROL PROGRAMME EFFECTIVENESS? COMPARING PERFORMANCE INDICATORS USING EVIDENCE SYNTHESIS TO ESTIMATE LOCAL INCIDENCE AND PREVALENCE FROM SURVEILLANCE DATA}

Joanna Lewis, Peter White. Imperial College London, London, UK

\subsection{6/sextrans-2017-053264.345}

Introduction Understanding patterns of chlamydia incidence and prevalence is important for addressing inequalities, planning cost-effective control programmes and defining performance indicators. Population-based surveys are costly; the best data for England come from the Natsal surveys which are only available once per decade, and are nationally representative but not powered to compare localities. Estimates at finer spatial and temporal scales are required.

Methods We present a method for estimating local incidence and prevalence by modelling the infection, testing and treatment processes. Parameters describing natural history and treatment-seeking behaviour are informed by the literature or calibrated using national prevalence estimates. By combining them with local-level surveillance data on numbers of chlamydia tests and diagnoses in England, we estimate local screening rates, incidence and prevalence.

Results There is substantial local-level variation in infection burden. Highest infection rates are in the most-deprived areas - but deprivation is a poor predictor of prevalence, with large variation within each deprivation quintile. Importantly, positivity is not a reliable proxy for prevalence. Most localities that meet the current performance target of 2300 annual diagnoses per 100000 population have higher incidence and prevalence than most that do not, and the target may be unrealistic for many localities.

Conclusion Our approach provides local estimates of chlamydia incidence and prevalence from surveillance data, which can be used to inform analysis of local variation and assess local control programmes. Many localities are unlikely to be able to meet the current annual diagnosis rate target, and successful prevention interventions like condom promotion make the target harder to reach. A better performance indicator could be the proportion of incident infections that are treated, as estimated by our model, since a higher value is always better for public health and other prevention activities make a higher value easier to achieve.

\section{P3.111 OPTIMISING STI SCREENING IN HIV-INFECTED MEN WHO HAVE SEX WITH MEN (MSM)}

Jodie Dionne-Odom, Kristal Aaron, Brice Daniels, Edward W Hook, Barbara Van Der Pol. University of Alabama at Birmingham, Birmingham, USA

\subsection{6/sextrans-2017-053264.346}

Introduction Current CDC guidelines recommend screening "at least annually" for Chlamydia trachomatis (CT) and Neisseria gonorrhoeae (GC) at sites of exposure using nucleic acid amplification tests (NAAT) in HIV-infected MSM. National screening rates remain suboptimal in this high-risk population, particularly at extra-genital sites.

Methods We enrolled HIV-infected MSM from a routine care visit at the $1917 \mathrm{HIV}$ clinic in Birmingham, Alabama. Inclusion criteria included age $>18$, receptive anal intercourse in the past 30 days and lack of antibiotic exposure. Participants provided four self-collected rectal swabs and a urine sample. A pharyngeal sample was provider-collected. Samples from the rectal and genital sites were run on four testing platforms with the composite infection standard ( $\geq 2$ NAAT positive) defining a positive result. Pharyngeal samples were run on two platforms and the patient infection standard (2 NAAT positive) was used to define positivity.

Results A total of 175 unique HIV-infected MSM were enrolled between December 2014 and November 2016. Overall, 34 men (19.4\%) had CT or GC infection detected. CT infection rates by site were: $13.1 \%$ rectal, $3.4 \%$ urogenital, $0 \%$ pharyngeal. GC infection rates by site were: $8.6 \%$ rectal, $3.4 \%$ urogenital and $2.3 \%$ pharyngeal. In addition, $5.7 \%$ of men had co-infection with CT and GC at the rectal site and $1.7 \%$ had simultaneous CT or GC infection at genital and rectal sites. Most infections (79.4\%) would have been missed by genital screening alone.

Conclusion Sexually active, HIV-infected MSM in Birmingham, Alabama have high prevalence rates of CT and GC infection, particularly at the rectal site. This has public health implications since CT/GC coinfection may increase HIV transmission rates. Clinics that provide care for HIV-infected MSM should streamline extragenital testing; this may include the incorporation of patient-collected rectal swabs into routine care. 